\title{
Multi Focus Image Fusion using Combined Median and Average Filter based Hybrid Stationary Wavelet Transform and Principal Component Analysis
}

\author{
Tian Lianfang \\ School of Automation Science and Engineering, South China \\ University of Technology; Research Institute of Modern \\ Industrial Innovation, South China University of Technology; \\ Key Laboratory of Autonomous Systems and Network Control \\ of Ministry of Education, Guangzhou, China
}

Jameel Ahmed Bhutto

School of Automation Science and Engineering, South China University of Technology, Guangzhou, China

\author{
Du Qiliang \\ School of Automation Science and Engineering, South China \\ University of Technology; Research Institute of Modern \\ Industrial Innovation, South China University of Technology; \\ Key Laboratory of Autonomous Systems and Network Control \\ of Ministry of Education, China \\ Bhawani Shankar \\ IEEE Senior member, Dean Faculty of Electrical, Electronic \\ and Computer Engineering, Mehran University of Engineering \\ and Technology, Jamshoro, Sindh, Pakistan
}

\author{
Saifullah Adnan \\ School of Electronic and Information Engineering, South China University of Technology, Guangzhou, China
}

\begin{abstract}
Poor illumination, less background contrast and blurring effects makes the medical, satellite and camera images difficult to visualize. Image fusion plays the vital role to enhance image quality by resolving the above issues and reducing the image quantity. The combination of spatial and spectral technique Discrete Wavelet Transform and Principal Component Analysis (DWT-PCA) decrease processing time and reduce number of dimensions but down sampling causes lack of shift invariance that results in poor quality final fused image. At first this work uses combined median and average filter that eliminates noise in the image which is caused by illumination, camera circuitry and sensor at preprocessing stage. Then, hybrid Stationary Wavelet Transform and Principal Component Analysis (SWT-PCA) technique is implemented to increase output image accuracy by eliminating down sampling and is not influenced by artifacts and blurring effects. Further, it can overcome the trade-off of Heisenberg's uncertainty principle by improving accuracy in both domains, time (spatial) as well as frequency (spectral). The proposed combined median and average filter with hybrid SWT-PCA algorithm measures quality parameters, such as peak signal to noise ratio (PSNR), mean squared error (MSE) and normalized cross correlation (NCC) and improved results depict the superiority of the algorithm than existing techniques.
\end{abstract}

Keywords - Image fusion; Heisenberg's uncertainty principle; combined median and average filter; Haar wavelet; Stationary Wavelet Transform and Principal Component Analysis (SWT-PCA)

\section{INTRODUCTION}

Image fusion is the process of extracting high quality, more informative single image out of multiple images by removing artifact, noise and blurring effects [1]-[3]. Image fusion is an interdisciplinary area of research and has received a lot of interest in academic, industrial, hospitals, manufacturing, robotics, military and computer vision [4], [5]. The spatial domain techniques such as PCA, averaging method have low spectral resolution whereas spectral domain such as DWT, Curve-let, SWT techniques have low spatial resolution therefore it degrades the quality of output image [6].

In [7], Moris has developed image fusion technique based on Maximum method in which blurring effects limit the contrast of fused image. Simple averaging method is used for image fusion in [8]. However, better quality image is not produced due to artifacts and noise issues. Spectral domain technique named Curve-let transform is implemented by Choi in [9]. It produces better results than discrete wavelet transform but it suffers performance degradation if image is not curved shape. A good data compression technique named PCA developed in [10] that reduces the number of dimensions, however it suffers spectral degradation. In [11], author has proposed a Discrete Wavelet transform technique that achieves better and precise results with fast computation but it suffers with spatial degradation that reduces the overall performance of output image. In [12], author has implemented the Discrete Stationary Wavelet transform that minimizes spectral degradation in comparison to DWT. The shortcoming of this technique is less spatial resolution. The DWT-PCA has been implemented in [13] to achieve better results in both domains, spatial and spectral. Though this technique achieves good quality image than existing algorithms, but the final fused image is still affected by shift-invariance due to down sampling. 
In this paper, combined median and average filter at preprocessing stage has been used. It does not only reduce the complexity but also preserve the edges by reducing the image noise. At the same time, statistical histogram is applied to improve the searching speed of median value. The proposed technique achieve fast computation by PCA and high quality image by eliminating down sampling in SWT. In addition, Haar wavelet is used in our work because it has number of attractive features such as, orthogonality, compact support, and infinite support in frequency domain, symmetric in scaling function and anti-symmetric in wavelet function. These all characteristics make this work more accurate, robust and memory efficient. This proposed method for multi-focus data finds applications in various fields such as recognition of objects, feature extraction in military surveillance as well as in aeronautical observations.

The rest of the paper is arranged as follows: Image fusion techniques and proposed hybrid model has been elaborated in Sections 2 and 3, respectively. Finally simulation results and conclusion will be discussed in Sections 4 and 5.

\section{TECHNIQUES OF IMAGE FUSION}

Image fusion techniques are classified in two domains; spatial (time) and Spectral (frequency) which are discussed below:

\section{A. $P C A$}

PCA transforms the number of correlated variables into uncorrelated variables that makes it more accurate and reliable. Moreover, it reduces the number of dimensions by choosing the highest eigenvalue vector as the principle component that results in fast computation [14]. It is quantitatively rigorous method that generates new set of variables called principal components and all these components are orthogonal to each other which mitigate redundant information. PCA is widely used in many applications such as image processing, machine learning, wireless communication, pattern matching and so on [7], [14]-[16].

The flow diagram for PCA technique is shown in Fig. 1. The input images are arranged in column vectors and their resulting vector has dimension of $n \times 2$ where $\mathrm{n}$ is length of each vector. The eigenvectors and eigenvalues are computed for both images. Finally the principle components $p c_{1}$ and $p c_{2}$ are obtained from eigenvectors corresponding to largest eigenvalue [16], [17].

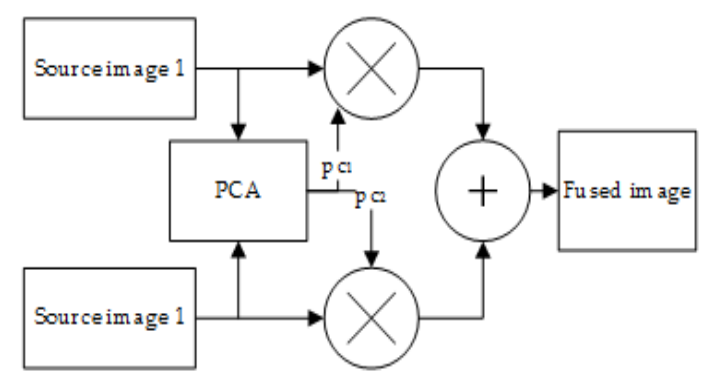

Fig. 1. Diagram of PCA.
The analysis of PCA involves five main steps:

- Produce the column vector from source images.

- Get covariance /correlation matrix $A$ from data sets of input images. Variance and covariance are obtained from data set of input images; $x_{1}$ and $x_{2}$.

$$
\begin{aligned}
& A=\left[\begin{array}{cc}
v_{1} & c_{\mathrm{var}}(1,2) \\
c_{\mathrm{var}}(1,2) & v_{2}
\end{array}\right] \\
& c_{\mathrm{var}}=\frac{\left(\sum\left(x_{1}-\overline{x_{1}}\right) \times\left(x_{2}-\overline{x_{2}}\right)\right)}{N-1}
\end{aligned}
$$

$v_{1}, v_{2}, c_{\mathrm{var}}(1,2)$ and $N$ represents the variance, covariance and number of terms respectively.

- The Eigen values can be obtained by solving:

$$
\operatorname{Det}|A-\lambda I|=0
$$

$\lambda$ represents the eigenvalues.

$$
\begin{aligned}
& {\left[\begin{array}{cc}
v_{1} & c_{\mathrm{var}}(1,2) \\
c_{\mathrm{var}}(1,2) & v_{2}
\end{array}\right]-\lambda I=0} \\
& \left(\left(v_{1}-\lambda\right) \times\left(v_{2}-\lambda\right)\right) \times c_{\mathrm{var}}(1,2)^{2}=0
\end{aligned}
$$

- Eigen vectors are solved as follow:

$$
|A-\lambda I| \times[X]=0
$$

- The co-ordinates of each data point in the direction of principal component are obtained by:

$$
p c_{j}=a_{i 1} Y_{1}+a_{i 2} Y_{2} \ldots \ldots \ldots a_{i n} Y_{n}
$$

Where

$a_{i}$ is coefficient of factor $i, p c_{j}$ is the $j_{t h}$ principle component and $Y_{1}, Y_{2} \ldots Y_{n}$ represents coordinates of each data.

\section{B. DWT}

It provides a compact representation of signal's frequency component by achieving better frequency information and good time resolution. It decomposes image into frequency subbands at different scale by splitting into high and low frequency [18]. The low frequency contains average intensity of image whereas high frequency provides edges information. Down sampling in DWT decreases the computation time and speed up the algorithm [13], [17]-[19].

It is depicted in Fig. 2, the DWT separately filters and down samples 2D image in horizontal and vertical direction. The source image is filtered by low pass (L) and high pass $(\mathrm{H})$ filter in horizontal direction and down-sampled by factor 2 to get the coefficients matrices $\operatorname{Im}(\mathrm{L})$ and $\operatorname{Im}(\mathrm{H})$. Same filters with down sampling are applied to coefficient matrices in vertical direction to obtain sub-bands, $\operatorname{Im}(\mathrm{LL}), \operatorname{Im}(\mathrm{LH})$, 
$\operatorname{Im}(\mathrm{HL})$ and $\operatorname{Im}(\mathrm{HH})$ that are images with low-low, low-high, high-low and high-high frequency respectively [15], [20].

The wavelet decomposition is given by following equation:

$$
\begin{aligned}
& G_{j+1}=H G_{j} H^{\prime} \\
& D_{j+1}^{h}=G G_{j} H^{\prime} \\
& D_{j+1}^{v}=H G_{j} G^{\prime} \\
& D_{j+1}^{d}=G G_{j} G^{\prime}
\end{aligned}
$$

Where $j=0,1,2, \ldots . j-1$ is for decomposition level. $\mathrm{H}$ for low-pass filtering; $\mathrm{G}$ for High-pass filtering; $H^{\prime}$ and $G^{\prime}$ are conjugate of the $\mathrm{H}$ and $\mathrm{G} ;{ }^{G_{j+1}}, D_{j+1}^{h}, D_{j+1}^{v}$ and $D_{j+1}^{d}$ are approximate, horizontal, vertical and diagonal details of images respectively.

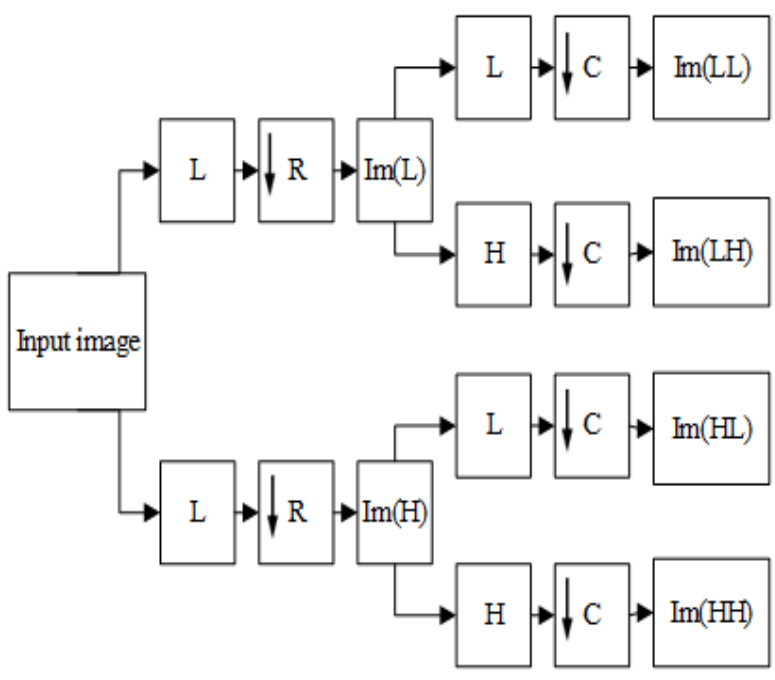

Fig. 2. Image decomposition by DWT.

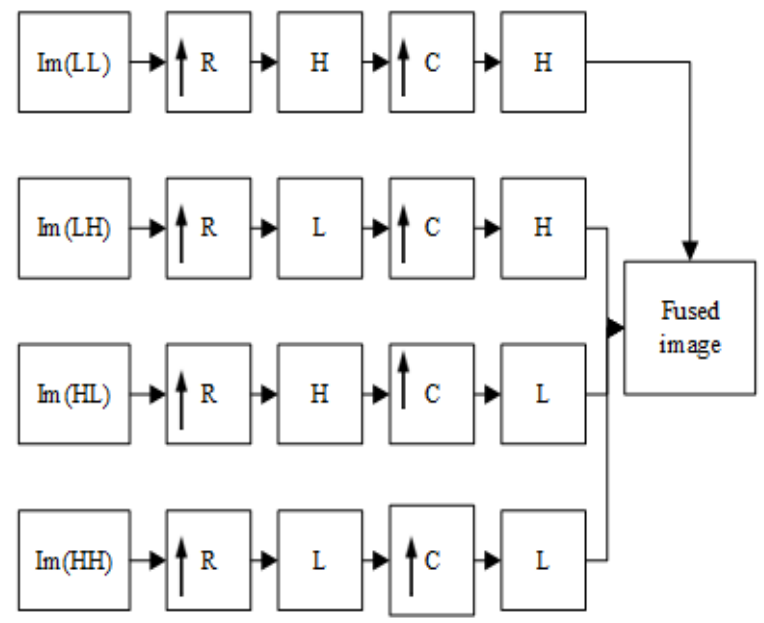

Fig. 3. Image reconstruction by DWT.
Inverse DWT is applied to reconstruct 2D image from subbands $\operatorname{Im}(\mathrm{LL}), \operatorname{Im}(\mathrm{LH}), \operatorname{Im}(\mathrm{HL})$ and $\operatorname{Im}(\mathrm{HH})$ as depicted in Fig. 3. This involves column up sampling the both; low and high pass filters for each sub-band images. Same filters are applied with row-up sampling to get the fused image. Image reconstruction equation is given as:

$$
G_{j}=H^{\prime} G_{j+1} H+G^{\prime} D_{j+1}^{h} H+H^{\prime} D_{j+1}^{v} G+G^{\prime} D_{j+1}^{d} G
$$

Here ${ }^{j=j-1, j-2, \ldots 0}, G_{j+1}$ represents low frequency component (approximate detail) and $D_{j+1}^{h}, D_{j+1}^{v}$ and $D_{j+1}^{d}$ denote high frequency components respectively (horizontal, vertical and diagonal detail).

\section{SWT}

Down sampling in DWT causes lack of shift-invariance that adds distortion, artifacts and blurring effects which results poor quality output image. These issues can be resolved by eliminating the down-sampling in SWT. Therefore, preserving a high quality and more informative output image [16], [21].

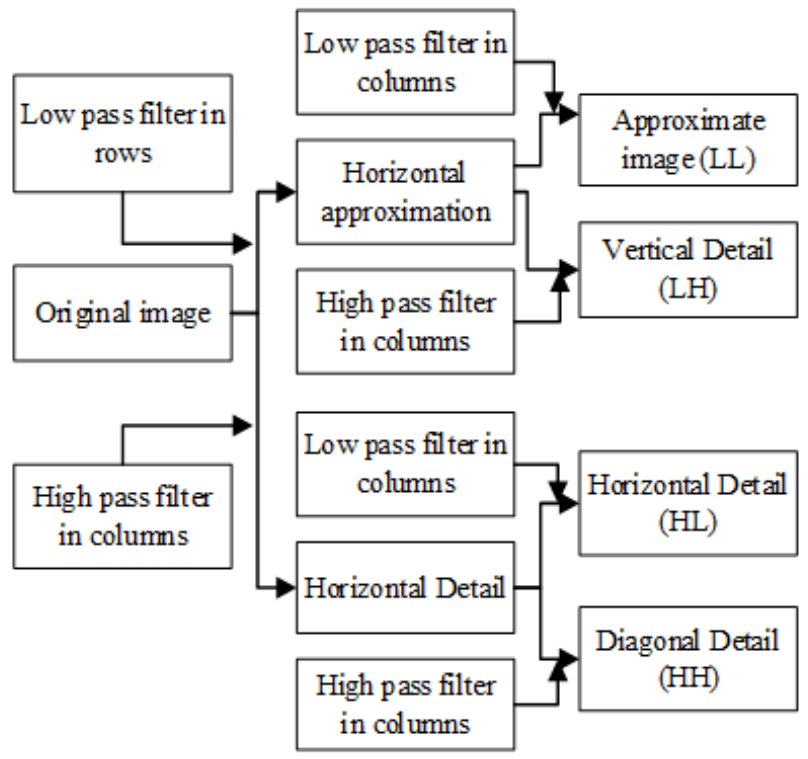

Fig. 4. Diagram of SWT.

The original image is decomposed into horizontal and vertical approximation by applying column wise and row wise low pass and high pass filters [21]. Same filtration is applied to decomposed parts in rows wise and column wise to obtain Approximate, vertical, horizontal and diagonal detail as elaborated in Fig. 4. The low pass and high pass filters preserve low and high frequencies and provides detailed information at respective frequencies.

The wavelet decomposition equation for SWT is given as:

$$
\begin{gathered}
A_{j}, k_{1}, k_{2}=\sum_{n 1} \sum_{n 2} h_{0}^{\uparrow 2 j}\left(n_{1}-2 k_{1}\right) h_{0}^{\uparrow 2 j}\left(n_{2}-2 k_{2}\right) A_{j-1}, n_{1}, n_{2}(13) \\
D_{j}^{1}, k_{1}, k_{2}=\sum_{n 1} \sum_{n 2} h_{0}^{\uparrow 2 j}\left(n_{1}-2 k_{1}\right) g_{0}^{\uparrow 2 j}\left(n_{2}-2 k_{2}\right) A_{j-1}, n_{1}, n_{2} \text { (14) }
\end{gathered}
$$




$$
\begin{aligned}
& D_{j}^{2}, k_{1}, k_{2}=\sum_{n 1} \sum_{n 2} g_{0}^{\uparrow^{22 j}}\left(n_{1}-2 k_{1}\right) h_{0}^{\uparrow 2 j}\left(n_{2}-2 k_{2}\right) A_{j-1}, n_{1}, n_{2} \\
& D_{j}^{3}, k_{1}, k_{2}=\sum_{n 1} \sum_{n 2} g_{0}^{\uparrow^{2 j}}\left(n_{1}-2 k_{1}\right) g_{0}^{\uparrow^{2 j}}\left(n_{2}-2 k_{2}\right) A_{j-1}, n_{1}, n_{2}
\end{aligned}
$$

Where $A_{j}, k_{1}, k_{2} ; D_{j}^{1}, k_{1}, k_{2} ; D_{j}^{2}, k_{1}, k_{2}$ and $D_{j}^{3}, k_{1}, k_{2}$ are low frequency (LL), horizontal high frequency $(\mathrm{LH})$, vertical high frequency (HL), and diagonal component (HH) respectively for SWT. $h_{0}^{\uparrow 2 j}, g_{0}^{\uparrow 2 j}$; indicate that $2^{j}-1$ zeros are added between $h_{0}$ and $g_{0}$.

$$
\begin{aligned}
A_{j-1}, n_{1}, n_{2}= & \frac{1}{4} \sum_{i=0}^{3}\left(\sum_{k_{1}} \sum_{k_{2}} h_{1}\left(n_{1}-2 k_{1}-i\right) h_{1}\left(n_{2}-2 k_{2}-i\right) A_{j}, k_{1}, k_{2}\right. \\
& +\sum_{k_{1}} \sum_{k_{2}} h_{1}\left(n_{1}-2 k_{1}-i\right) g_{1}\left(n_{2}-2 k_{2}-i\right) D_{j}^{1}, k_{1}, k_{2} \\
& +\sum_{k_{1}} \sum_{k_{2}} g_{1}\left(n_{1}-2 k_{1}-i\right) h_{1}\left(n_{2}-2 k_{2}-i\right) D_{j}^{1}, k_{1}, k_{2} \\
& \left.+\sum_{k_{1}} \sum_{k_{2}} g_{1}\left(n_{1}-2 k_{1}-i\right) g_{1}\left(n_{2}-2 k_{2}-i\right) D_{j}^{2}, k_{1}, k_{2}\right)
\end{aligned}
$$

The SWT can be reconstructed by (17) shown above.

\section{Combined Median and Average Filter}

The image noise is random variation that is produced by illumination, sensor and camera circuitry. It does not only add distortion in image but highly affects the visual effects. This noise can be reduced by a non-linear median filter that preserves the edges and sharpen the contrast as well. The noise reducing effects depend on the shape and size of filtering mask whereas algorithm complexity depends on searching speed to get the median value. Authors have proposed fast algorithms that improve the searching speed of median filter and reduced the complexity while preserves the edges without being affected by noise [22].

The combined median and average filter achieves better performance for noise reduction that adaptively resizes the mask filter according to level of noise mask. This technique reduces the noise and retain the better image detail by preserving the edges. We sequentially check each pixel for noise reduction. If the pixel value is greater than the average value, it means the pixel is affected by noise and we replace that pixel with median value of the mask; otherwise, we unchange that value of the pixel [22].

\section{PROPOSED AlgorithM}

We have implemented a combined median and average filter based hybrid SWT-PCA which is discussed briefly.

\section{A. Combined Median and Average Filter based Hybrid SWT- PCA Algorithm}

The proposed method involves two steps: At preprocessing stage, combined median and average filter is applied on input images then image fusion is done by hybrid SWT-PCA. Prepressing stage involves the following steps:

Step 1: Adaptively resize the mask

- Initialize the filter by $n=3$
- Compute $A_{1}=$ med-min; $A_{2}=$ med $-\max$

- Verify, if $A_{1} \succ 0$ and $A_{2} \prec 0$ then return to step 1 otherwise enlarge the mask size by $n=n+2$.

Where ${ }^{\text {med }}$, min , med $A_{1}$ and $n$ represent the median, minimum, maximum, average values and mask size, respectively.

Step 2: Apply median filtering to find median values.

The output images $O_{1}$ and $O_{2}$ are obtained by applying filters to the input images. Furthermore, using hybrid SWTPCA, $O_{1}$ and $O_{2}$ images are decomposed into four sub bands $L L_{1}, L H_{1}, H L_{1}, H H_{1}$ and $L L_{2}, L H_{2}, H L_{2}, H H_{2}$ by SWT. We compute the PCA for these sub band and eigen vectors with maximum values are selected. Each sub band images are multiplied and summed to combine mentioned sub bands. The new sub-bands $L L_{\text {new }}, L H_{\text {new }}, H L_{\text {new }}$ and $H H_{\text {new }}$ are calculated by following equations:

$$
\begin{aligned}
& L L_{\text {new }}=p c_{1} \times L L_{1}+p c_{2} \times L L_{2} \\
& L H_{\text {new }}=p c_{3} \times L H_{1}+p c_{4} \times L H_{2} \\
& H L_{\text {new }}=p c_{5} \times H L_{1}+p c_{6} \times H L_{2} \\
& H H_{\text {new }}=p c_{7} \times H H_{1}+p c_{8} \times H H_{2}
\end{aligned}
$$$$
p c_{1}, p c_{2} \ldots \ldots . . p c_{8} \text { are principal components for sub bands, }
$$
respectively.

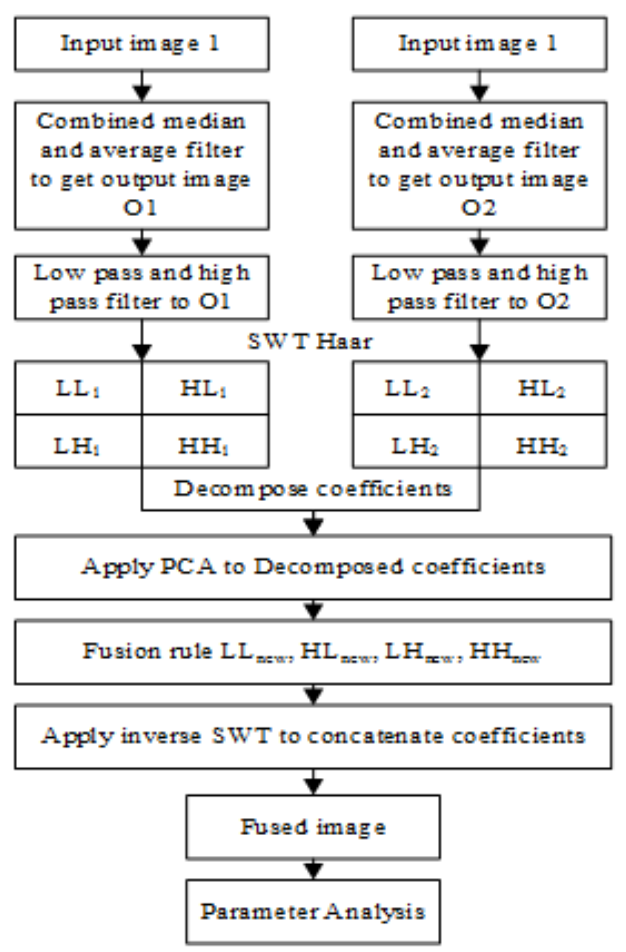

Fig. 5. Proposed SWT-PCA algorithm. 
The proposed hybrid SWT-PCA algorithm is depicted in Fig. 5. Combined median and average filter is applied to input images. The output of this filter is feed to low pass and high pass filters in row wise and column wise. The decomposed sub-bands $L L_{1}, L H_{1}, H L_{1}, H H_{1}$ and $L L_{2}, L H_{2}, H L_{2}, H H_{2}$ are obtained by applying the Haar wavelet. SWT does not use down sampling that makes the fusion output stable and consistent with original input sequence. Resulting coefficients are evaluated by PCA and each coefficient of source image is applied to corresponding coefficient of PCA. Fusion rule merges the PCA components with new decomposed coefficient ( $L L_{\text {new }}, L H_{\text {new }}, H L_{\text {new }}$ and $H H_{\text {new }}$ ). Finally, more informative and high quality fused image is obtained by applying inverse SWT on coefficients of combined transform.

The flowchart of combined median and average filter based SWT-PCA technique is shown in Fig. 6.

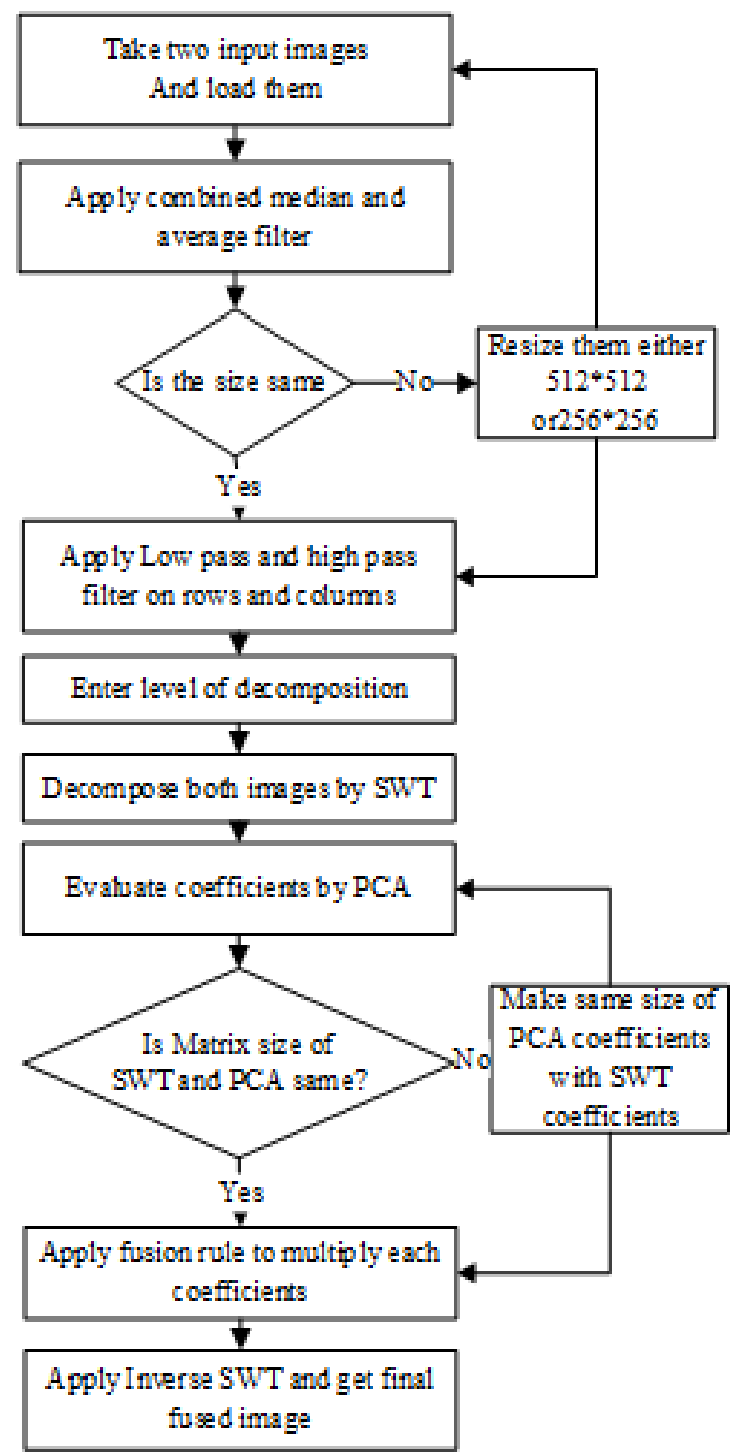

Fig. 6. Flowchart of proposed algorithm.

\section{Simulation Results AND Discussion}

In this paper, we have used MATLAB tool for our simulation and the images have been taken from the website http://imagefusion.org and paper [5].

\section{A. Simulation Results}

The simulation results given below show the comparison among DWT, SWT, DWT-PCA and combined median and average filter based SWT-PCA and their performance is measured on different quality parameters; PSNR, MSE and NCC.

\section{1) Scenario-01}

It can be seen from Fig. 7 that source images with different foci are combined by different image fusion techniques to produce better output image. Though DWT-PCA achieves better results than individual DWT and SWT but it is still affected by random variation in image. The proposed SWTPCA with combined median and average filter achieves better results than all existing fusion techniques without being affected by noise or random variation. Furthermore, it can be analyzed from Table I that there is only a little improvement of PSNR among DWT, SWT and DWT-PCA. Consequently, in proposed technique, PSNR jumps from 40.321 to 45.154 that shows its superiority.

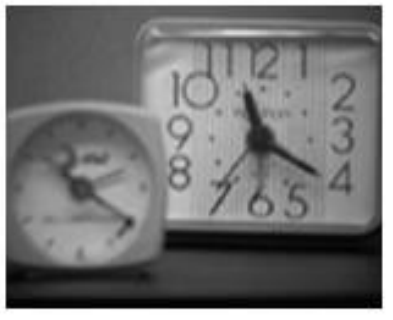

(a)

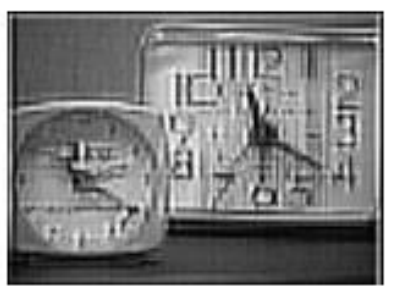

(c)

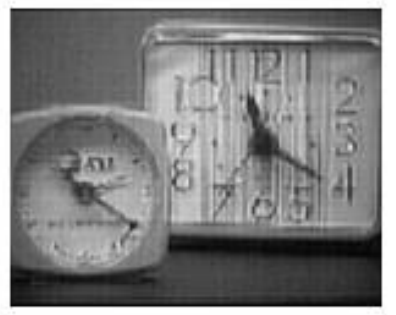

(e)

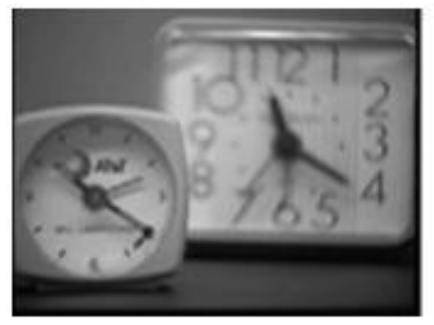

(b)

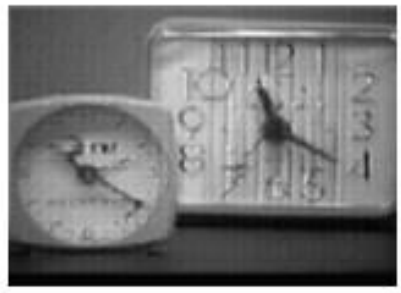

(d)

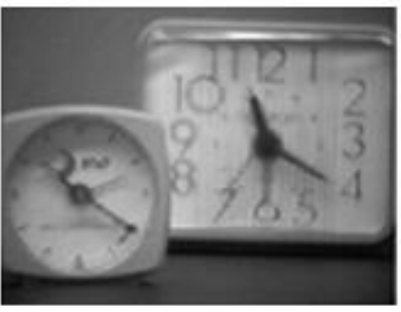

(f)
Fig. 7. The input focused images and output fused images: (a) Right focused input image; (b) Left focused input image; (c) DWT fused image; (d) SWT fused image; (e) DWT-PCA fused image; (f) Proposed method (SWT-PCA fused image). 
TABLE I. COMPARISON ANALYSIS OF DIFFERENT IMAGE FUSION TECHNIQUES

\begin{tabular}{|l|l|l|l|l|}
\hline & DWT & SWT & DWT-PCA & $\begin{array}{l}\text { Proposed } \\
\text { method }\end{array}$ \\
\hline PSNR & 36.732 & 38.297 & 40.321 & 45.154 \\
\hline MSE & 3.83 & 3.76 & 3.42 & 3.02 \\
\hline NCC & 0.35 & 0.41 & 0.47 & 0.57 \\
\hline
\end{tabular}

2) Scenario-02

Background focused and rope focused images are shown in Fig. 8. It can be clearly seen from Table II that PSNR of proposed technique jumps from 34.321 to 40.154 that depicts significant improvement. Similarly, MSE of proposed method reduces from 3.73 to 3.22 which shows better improvement as compared to existing techniques.

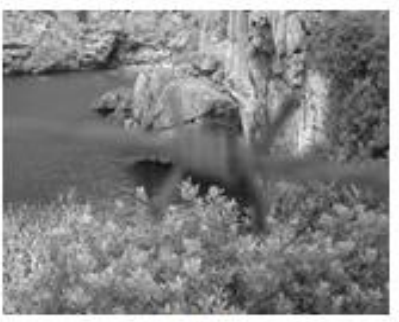

(a)

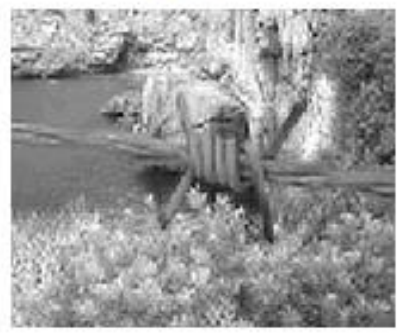

(c)

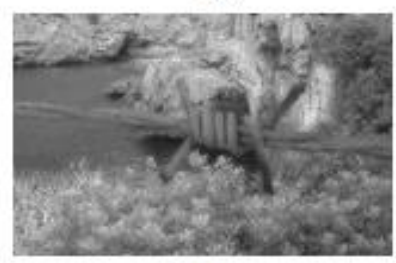

(e)

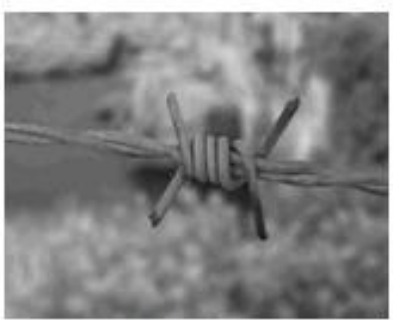

(b)

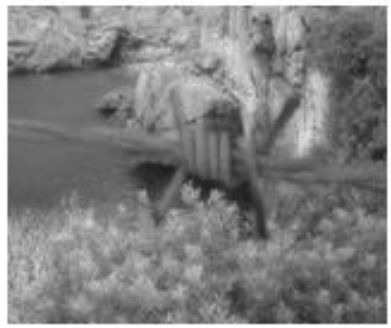

(d)

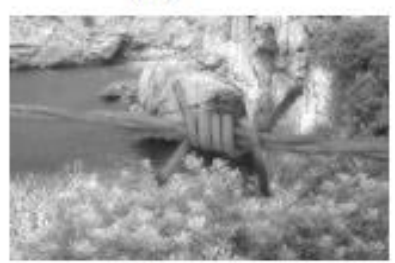

(f)
Fig. 8. The input focused images and output fused images: (a) Background focused input image; (b) Rope focused input image; (c) DWT fused image; (d) SWT fused image; (e) DWT-PCA fused image; (f) Proposed method (SWT-PCA fused image).

TABLE II. COMPARISON ANALYSIS OF DIFFERENT IMAGE FUSION TECHNIQUES

\begin{tabular}{|l|l|l|l|l|}
\hline & DWT & SWT & DWT-PCA & $\begin{array}{l}\text { Proposed } \\
\text { method }\end{array}$ \\
\hline PSNR & 31.432 & 32.597 & 34.321 & 40.154 \\
\hline MSE & 4.24 & 4.01 & 3.73 & 3.22 \\
\hline NCC & 0.28 & 0.37 & 0.43 & 0.54 \\
\hline
\end{tabular}

\section{3) Scenario-03}

It is shown in Fig. 9 that output fused image of DWT, SWT, DWT-PCA produce poor quality output image, whereas proposed method (SWT-PCA with combined median and average filter) eliminates the noise effect and produce better quality output image. Furthermore, it can be analyzed from Table III that there is less PSNR and NCC improvement in existing DWT, SWT and DWT-PCA. The proposed method achieves better improvement in PSNR and NCC with more reduction in MSE value. This shows the effectiveness of combined median and average filter with SWT and PCA.

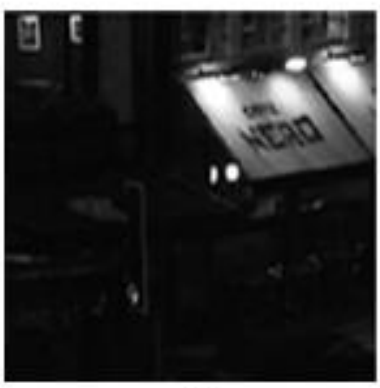

(a)

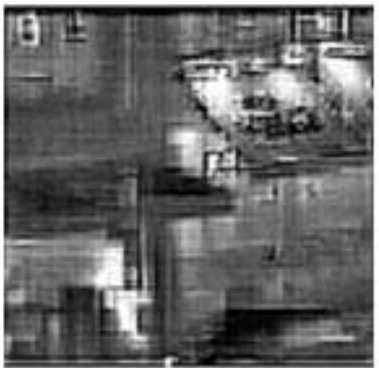

(c)

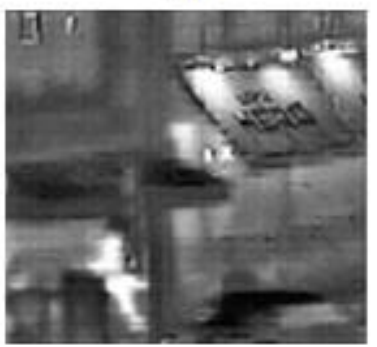

(e)

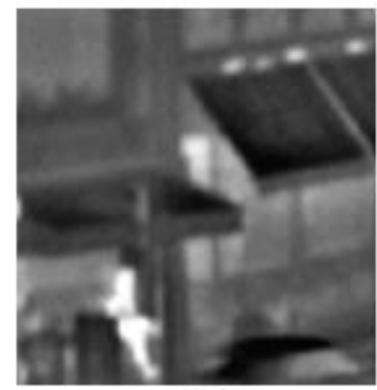

(b)

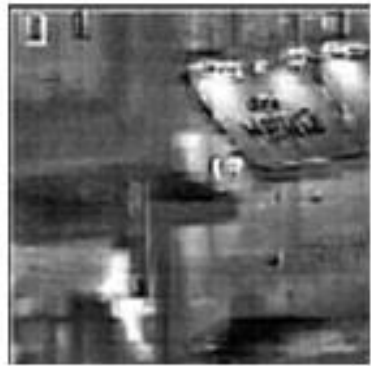

(d)

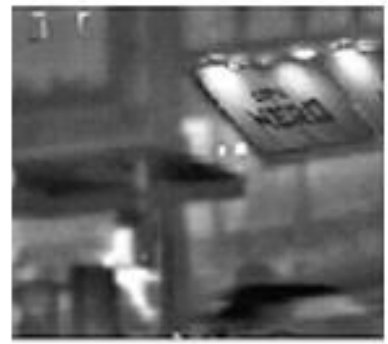

(f)
Fig. 9. The input focused images and output fused images: (a) Character focused input image; (b) Background focused input image; (c) DWT fused image; (d) SWT fused image; (e) DWT-PCA fused image; (f) Proposed method (SWT-PCA fused image).

TABLE III. COMPARISON ANALYSIS OF DIFFERENT IMAGE FUSION TECHNIQUES

\begin{tabular}{|l|l|l|l|l|}
\hline & DWT & SWT & DWT-PCA & $\begin{array}{l}\text { Proposed } \\
\text { method }\end{array}$ \\
\hline PSNR & 35.621 & 36.78 & 38.21 & 44.10 \\
\hline MSE & 3.21 & 3.04 & 2.87 & 2.14 \\
\hline NCC & 0.43 & 0.51 & 0.56 & 0.68 \\
\hline
\end{tabular}




\section{4) Scenario-04}

Fig. 10 shows that input images are affected by noise due to camera circuitry. The output fused image of DWT, DSWT, DWT-PCA is of poor quality due to noise. Proposed method eliminates the noise effect, produces better quality output image and achieves higher PSNR and NCC with less MSE than existing fusion techniques as shown in Table IV.

PSNR, MSE and NCC have been used as evaluation criteria in this paper. Higher the value of PSNR and NCC; better will be the final fused image. Similarly lower the MSE value corresponds to better output image. It can be clearly seen from above results that proposed technique (combined median and average filter based SWT-PCA) outperforms than current techniques by achieving lower MSE, higher PSNR and NCC values. Furthermore, it can be depicted from above figures that our proposed method remove the noise from input by combined median and average filter and obtain better accuracy by hybrid SWT-PCA.

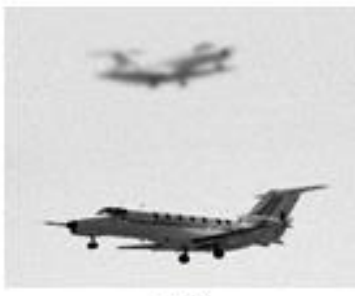

(a)

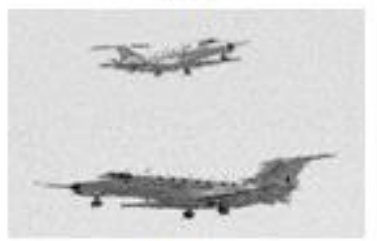

(c)

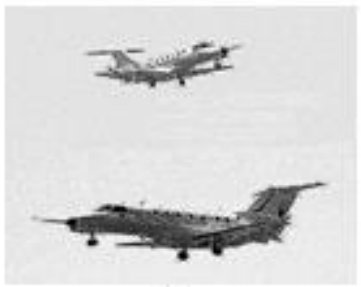

(e)

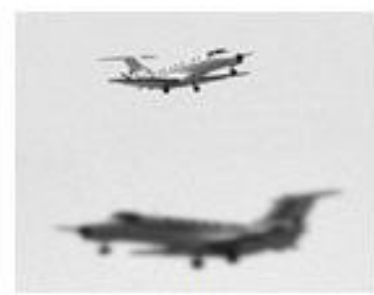

(b)

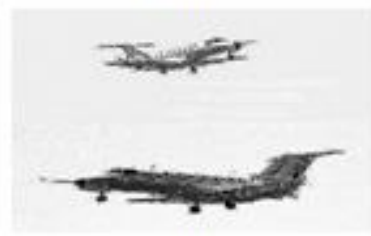

(d)

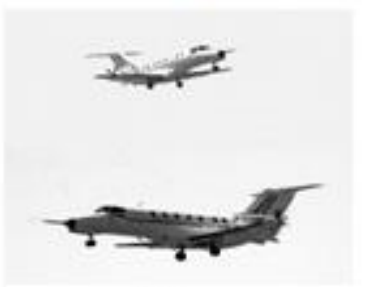

(f)
Fig. 10. The input focused images and output fused images: (a) Bottom focused input image; (b) Top focused input image; (c) DWT Fused image; (d) SWT fused image; (e) DWT-PCA fused image; (f) Proposed method (SWTPCA Fused image).

TABLE IV. COMPARISON ANALYSIS OF DIFFERENT IMAGE FuSION TECHNIQUES

\begin{tabular}{|l|l|l|l|c|}
\hline & DWT & SWT & DWT-PCA & $\begin{array}{l}\text { Proposed } \\
\text { method }\end{array}$ \\
\hline PSNR & 32.61 & 35.38 & 37.15 & 44.24 \\
\hline MSE & 4.53 & 4.21 & 4.02 & 3.26 \\
\hline NCC & 0.31 & 0.39 & 0.44 & 0.51 \\
\hline
\end{tabular}

\section{CONCLUSION}

Recently, image fusion techniques are being considered as the most prominent and has been used in many areas such as images for medical diagnosis, military and law enforcement, robotics, manufacturing, computer vision and so on. A lot of research has been carried out regarding improving the image quality but it suffers with issues like noise, artifacts, blurring effects, lack of shift-invariance and Heisenberg uncertainty principle. However, the simulation results of proposed algorithm for multi-focus images resolve the mentioned issues and evaluate the performance on the basis of PSNR, MSE and NCC. Lower values of MSE, higher values of PSNR and NCC show the superiority of proposed algorithm than existing conventional and combined DWT-PCA techniques. Combined median and average filtering with Haar wavelet in SWT-PCA excludes down sampling that makes the hybrid algorithm shift invariant and robust while preserving the image data by reducing noise and complexity. Triple modality is also hot research topic for future work and this idea can be implemented for opaque and semi-transparent images.

\section{ACKNOWLEDGMENT}

This research work is supported by:

The Guangzhou Research Project "Development of Intelligent Escalator System with Automatic Identification Ability" (201604010114).

2016 Provincial Specialized Fund for Frontier and Key Technical Innovation "R\&D and Application Demonstration of Fully Automated Equipping Production Line after Aluminum Extrusion Process" (2016B090912001).

National Ministry of Science and Technology Hai Phong Public Welfare Project "Sea Vessel Star-Machine-Island Stereoscopic Surveillance and Monitoring Technology System" (201505002).

\section{REFERENCES}

[1] A. Dogra, B. Goyal, and S. Agrawal, "From multi-scale decomposition to non-multi-scale decomposition methods: A comprehensive survey of image fusion techniques and its applications," IEEE Access, vol. 5, pp. 16040-16067, 2017.

[2] J. Luo and W. Kong, "The Infrared and Visible Light Image Fusion Based on the Non-subsample Shearlet Transform and Heat Source Concentration Ratio," in 2016 International Conference on Intelligent Networking and Collaborative Systems (INCoS), 2016, pp. 544-547.

[3] L. N. H. Hoa, L. D. Cuong, and L. C. Ke, "Enhanced spatial resolution for VNREDSat-1 multispectral images using IHS fusion technique based on sensor spectral response function," in 2016 Eighth International Conference on Knowledge and Systems Engineering (KSE), 2016, pp. 304-308.

[4] A. Galande and R. Patil, "The art of medical image fusion: A survey," in 2013 International Conference on Advances in Computing, Communications and Informatics (ICACCI), 2013, pp. 400-405.

[5] L. Jian, X. Yang, Z. Zhou, K. Zhou, and K. Liu, "Multi-scale image fusion through rolling guidance filter," Future Generation Computer Systems, vol. 83, pp. 310-325, 2018/06/01/ 2018.

[6] P. Kaur and M. Kaur, "A comparative study of various digital image fusion techniques: A review," International Journal of Computer Applications, vol. 114, 2015.

[7] C. Morris and R. S. Rajesh, "Two Stage Spatial Domain Image Fusion Techniques," ICTACT Journal On Image And Video Processing: Special Issue On Video Processing For Multimedia Systems, vol. 5, 2014. 
[8] A. Malviya and S. G. Bhirud, "Image fusion of digital images," Entropy, vol. 7, pp. 7-4955, 2009.

[9] M. Choi, R. Y. Kim, and M.-G. Kim, "The curvelet transform for image fusion," International Society for Photogrammetry and Remote Sensing, ISPRS 2004, vol. 35, pp. 59-64, 2004.

[10] A. Purushotham, G. U. Rani, and S. Naik, "Image fusion using DWT \& PCA," International Journal of Advanced Research in Computer Science and Software Engineering, vol. 5, pp. 800-4, 2015.

[11] D. Mistry and A. Banerjee, "Discrete wavelet transform using matlab," International Journal Of Computer Engineering \& Technology, vol. 4, pp. 252-259, 2013.

[12] M. Pradnya and S. D. Ruikar, "Image fusion based on stationary wavelet transform," International journal of Advanced Engineering Research and Studies, II/IV/July-Sept, pp. 99-101, 2013.

[13] S. Mane and S. D. Sawant, "Image fusion of CT/MRI using DWT, PCA methods and analog DSP processor," Int. Journal of Engineering Research and Applications, vol. 4, pp. 557-563, 2014.

[14] M. S. A. M. a. N. K. a. T. A. a. M. A. a. F. R. a. M. Awais, "Pakistan Sign Language Detection using PCA and KNN," International Journal of Advanced Computer Science and Applications, vol. 9, 2018.

[15] S. A. Panwar and S. Malwadkar, "A Review: Image Fusion Techniques for Multisensor Images," ed: IJAREEIE, 2015.
[16] S. Baraiya and L. P. Gagnani, "An introduction of image fusion techniques," International Journal for Innovative Research in Science and Technology, vol. 1, pp. 86-89, 2014.

[17] S. S. Bedi, J. Agarwal, and P. Agarwal, "Image fusion techniques and quality assessment parameters for clinical diagnosis: a review," International journal of advanced research in computer and communication engineering, vol. 2, pp. 2319-5940, 2013.

[18] V. T. H. T. a. N. T. Binh, "Object Contour in Low Quality Medical Images in Curvelet Domain," International Journal of Advanced Computer Science and Applications, vol. 9, 2018.

[19] X. Zhou, H. Zhang, and C. Wang, "A Robust Image Watermarking Technique Based on DWT, APDCBT, and SVD," Symmetry, vol. 10, 2018.

[20] K. Harpreet and R. Rachna, "A combined approach using DWT \& PCA on image fusion," Int J Adv Res Comput Commun Eng, vol. 4, pp. 294296, 2015.

[21] S. K. Shah and D. U. Shah, "Comparative study of image fusion techniques based on spatial and transform domain," International Journal of Innovative Research in Science, Engineering and Technology (IJIRSET), vol. 3, 2014.

[22] Y. Zhu and C. Huang, "An Improved Median Filtering Algorithm for Image Noise Reduction," Physics Procedia, vol. 25, pp. 609-616, 2012/01/01/ 2012. 